\title{
Efficacy of on-demand intrahepatic arterial therapy in combination with sorafenib for advanced hepatocellular carcinoma
}

This article was published in the following Dove Medical Press journal: OncoTargets and Therapy

\section{Kazuto Tajiri \\ Yuka Futsukaichi \\ Saito Kobayashi \\ Kohei Nagata \\ Satoshi Yasumura \\ Terumi Takahara \\ Masami Minemura \\ Ichiro Yasuda}

Department of Gastroenterology, Toyama University Hospital,

Toyama, Japan
Correspondence: Kazuto Tajiri Department of Gastroenterology, Toyama University Hospital, 2630 Sugitani, Toyama 930-0194, Japan

$\mathrm{Tel}+81764347301$

Fax +8I 764345027

Email tajikazu@med.u-toyama.ac.jp
Purpose: The purpose of this study was to evaluate the effectiveness and tolerability of "on-demand" combination therapy with sorafenib and hepatic arterial treatments, such as transarterial chemoembolization and hepatic arterial infusion chemotherapy, in patients with hepatocellular carcinoma (HCC).

Patients and methods: Eighty consecutive patients with advanced HCC, 58 administered sorafenib monotherapy and 22 administered on-demand combination therapy, were retrospectively evaluated.

Results: The disease control rate was significantly higher in the combination group than in the monotherapy group ( $86.3 \%$ vs $51.7 \%, p=0.01)$. Elevated alanine aminotransferase levels were significantly more frequent in the combination group $(40.9 \%$ vs $12.1 \%, p=0.01)$, but it was tolerable. Progression-free survival ( 180 vs 45 days, $p=0.045$ ) and overall survival ( 983 vs 452 days, $p=0.004$ ) were significantly longer in the combination group, as was the duration of sorafenib treatment (367 vs 66 days, $p<0.001$ ). Multivariate analysis showed that hepatitis $\mathrm{C}$ virus infection, disease control, and combination therapy were positive independent prognostic factors for survival, whereas alpha-fetoprotein $>400 \mathrm{ng} / \mathrm{mL}$ was negatively prognostic. In patients receiving combination therapy, male sex, hepatitis B virus infection, performance status deterioration, Barcelona clinic liver cancer-B, and major vascular invasion were prognostic of survival.

Conclusion: On-demand combination therapy was tolerated and may be a therapeutic option for patients with advanced HCC.

Keywords: hepatocellular carcinoma, sorafenib, combination therapy, transarterial chemoembolization, hepatic arterial infusion chemotherapy

\section{Introduction}

Hepatocellular carcinoma (HCC) is a life-threatening disease worldwide and frequently diagnosed at an advanced stage. ${ }^{1,2}$ Therefore, most patients are ineligible for curative treatments, such as resection, locoregional ablative therapy, and liver transplantation. ${ }^{3}$ Survival of patients with unresectable HCC may be prolonged by transarterial chemoembolization (TACE), hepatic arterial infusion chemotherapy (HAIC), or systemic therapy. TACE is a standard treatment option for patients with multiple HCC nodules located only in the liver and not infiltrating major blood vessels (intermediate stage, Barcelona clinic liver cancer [BCLC]-B). ${ }^{4-6}$ Treatment with the oral multikinase inhibitor sorafenib may provide better prognosis for patients with BCLC-B HCC who are refractory to TACE, ${ }^{7,8}$ and significantly improve survival in patients with advanced HCC accompanied by major vascular invasion (MVI) or 
extrahepatic metastasis (EHM; advanced stage, BCLC-C). ${ }^{9,10}$ Another oral multikinase inhibitor, regorafenib, was recently shown to improve survival in patients with advanced HCC who were refractory to sorafenib (RESORCE trial), leading to its approval as a second-line treatment for advanced HCC. ${ }^{11}$ Furthermore, lenvatinib has been shown to be effective similar to sorafenib as first-line therapy in patients with advanced HCC. ${ }^{12}$ Thus, at present, tyrosine-kinase inhibitors (TKIs) are standard treatments for patients with advanced, unresectable HCC.

Although shown to provide survival benefits in patients who achieve stable disease, sorafenib has an objective response rate, defined as the percentage of patients who show a reduction in tumor size, of only $2.0 \%-3.3 \%$, which is considered as unsatisfactory. ${ }^{9,10}$ Thus, combinations of sorafenib with other treatment modalities, especially TACE, have been tested. TACE has been shown to enhance the expression of vascular endothelial growth factor (VEGF), which is associated with the tumor growth, whereas sorafenib was found to suppress VEGF expression, leading to some favorable synergistic effects. ${ }^{13}$ Indeed, meta-analyses have shown that sorafenib combined with TACE improved the overall survival (OS), time to progression (TTP), and objective response ratio compared with TACE alone. ${ }^{14-17}$ In contrast, other meta-analyses showed that this combination improved TTP but not OS. ${ }^{18,19}$ Thus, combination therapy with sorafenib and TACE is theoretically promising, but its clinical benefits remain unclear, especially in patients refractory to TACE. In addition, HAIC has been shown to induce tumor shrinkage and regression of vascular invasion, especially in Japanese patients. The survival benefits of HAIC plus sorafenib have not yet been determined, although this combination has shown some favorable effects, especially in patients with major portal invasion. ${ }^{20-22}$

The present study retrospectively compared the efficacy and safety of sorafenib monotherapy and sorafenib plus on-demand TACE/HAIC in patients with advanced HCC. The results suggested that, compared with sorafenib alone, on-demand hepatic arterial treatments such as TACE or HAIC combined with sorafenib improved disease control and prolonged OS in patients with advanced HCC.

\section{Patients and methods}

\section{Patients}

The data of 80 consecutive patients with advanced stage HCC at Toyama University Hospital who were administered sorafenib (Nexavar ${ }^{\circledR}$; Bayer HealthCare Pharmaceuticals, West Haven, CT, USA) or were refractory to TACE from February 2009 to March 2017 were retrospectively reviewed. HCC was diagnosed based on typical radiological findings, increases in tumor markers, or histological findings. MVI and EHM were evaluated in all patients by computed tomography (CT), magnetic resonance imaging (MRI), and/or radioisotope examination before sorafenib treatment. In this study, MVI was defined as Vp3, Vp4, or Vv3. The presence of anti-hepatitis $\mathrm{C}$ virus (HCV) antibody was defined as positive for $\mathrm{HCV}$ infection, and the presence of hepatitis B surface antigen was defined as positive for hepatitis B virus (HBV) infection. Other etiologic causes of liver injury, including alcoholic and nonalcoholic fatty liver diseases and/or autoimmune liver diseases, were defined as "Others". Most patients had preserved liver function, defined at Child-Pugh grade A in this study, although some had Child-Pugh grade B (7 points). Albumin-bilirubin (ALBI) grade, a useful assessment of hepatic function for therapeutic decision making, was calculated as ( $\log 10$ bilirubin $[\mu \mathrm{mol} / \mathrm{L}] \times 0.66)+($ albumin $[\mathrm{g} / \mathrm{L}] \times-0.085)$, with grades 1,2 , and 3 defined as $\leq-2.60,>-2.60$ to -1.39 , and $>-1.39$, respectively. ${ }^{23,24}$ The BCLC-B stage was subclassified as described. ${ }^{25}$ Written informed consent was obtained from all participants or their proxies before treatment, and the study protocol was approved by the ethics committee of the University of Toyama (Approval Number: 25-31). This study was conducted in accordance with the Declaration of Helsinki.

\section{Sorafenib treatment}

Most patients were administered $800 \mathrm{mg}$ /day oral sorafenib (400 mg BID), although several patients with poor hepatic reserve function and performance status (PS) were started at $400 \mathrm{mg} /$ day at the discretion of their physician. Sorafenib treatment was suspended in patients with grades 3-4 hematological toxicity or grade 3 non-hematological toxicity but resumed at a reduced dose $(400 \mathrm{mg} /$ day $)$ when the toxicities improved to grade 1 or 2 . When required, the sorafenib dose was reduced to $400 \mathrm{mg}$ every other day. If further dose reduction was needed, administration of sorafenib was discontinued. Sorafenib was continued until each physician judged it ineffective or intolerable.

\section{Combination therapy}

Since 2013, patients with HCC at our institution who were deemed unlikely to achieve stable disease in response to sorafenib monotherapy, as shown by early radiological findings or changes in tumor markers within 4 weeks of sorafenib administration, or who had factors associated with tumor 
progression, such as MVI, were considered candidates for sorafenib plus TACE or HAIC. TACE plus sorafenib was administered to patients with progressive intrahepatic lesions (tumors that were rapidly growing and/or $>6 \mathrm{~cm}$ in diameter) at the discretion of the physician. In TACE treatment, angiography was performed by inserting a 3 Fr catheter through the femoral artery. The tip of the microcatheter was superselected into the tumor-feeding branches. After identification of the tumor-feeding artery, chemoembolization was achieved as selectively as possible with $1-7 \mathrm{~mL}$ of an emulsion of Lipiodol containing an anticancer agent (Miriplatin ${ }^{\circledR}$; Sumitomo Dainippon Pharma Co., Ltd., Osaka, Japan). Embolization with gelatin particles (Gerpart ${ }^{\circledR}$; Nippon Kayaku Co., Ltd; Tokyo, Japan) was performed after administration of the agent, until no tumor staining was observed on repeated angiography. Some patients refractory or intolerant to TACE with Miriplatin ${ }^{\circledR}$ underwent TACE using drug-eluting beads and Epirubicin ${ }^{\circledR}$ (epirubicin hydrochloride; Nippon Kayaku Co., Ltd., Tokyo, Japan). Sorafenib was continued during the TACE procedure. TACE was repeated every 8-12 weeks if residual viable tumor was evident, and TACE could be associated with good prognosis. Patients with MVI (Vp3 or $\mathrm{Vp} 4$ ) or refractory to TACE were treated with HAIC plus sorafenib. ${ }^{26}$ HAIC was performed using 5-fluorouracil (5-FU ${ }^{\circledR}$; Kyowa Hakko Kirin Co., Ltd. Tokyo, Japan) and IA call ${ }^{\circledR}$ (arterial cisplatin; Nippon Kayaku Co., Ltd.) as described. ${ }^{27}$ In patients who underwent implantation of a subcutaneous injection port for HAIC, sorafenib was discontinued for several days during the procedure but resumed as soon as possible. Combination therapy was continued until the physician judged it ineffective or intolerable. Combination therapy for intrahepatic lesions was administered to patients with extrahepatic metastases, if the former were considered prognostic of survival outcomes.

\section{Tumor response and toxicity assessment}

Tumor response was evaluated by dynamic CT or MRI, performed every 8-12 weeks, using the modified Response Evaluation Criteria in Solid Tumors. ${ }^{28}$ The best tumor response at each examination was documented. Assessment of adverse events found during treatment was based on National Cancer Institute Common Toxicity Criteria for Adverse Events version 4.0.

\section{Statistical analyses}

All statistical analyses were performed using SPSS version 19.0 (IBM Corporation, Armonk, NY, USA). Categorical variables were evaluated using the chi-square test or Fisher's exact test, as appropriate. Continuous variables were analyzed using the Mann-Whitney $U$ test. Progression-free survival (PFS) and OS after the start of sorafenib administration were analyzed using the Kaplan-Meier method and compared by log-rank tests. Univariate and multivariate analyses were performed using the Cox proportional hazards model. A $p$-value $<0.05$ was considered as statistically significant.

\section{Results \\ Patient characteristics}

The demographic and clinical characteristics of all 80 patients included in this study are given in Table 1. Most patients were men, with a median age of 68 years. The main causes of $\mathrm{HCC}$ were chronic $\mathrm{HCV}$ and $\mathrm{HBV}$ infection, with the major cause among the patients with nonviral etiology being alcohol (23/32). Liver function was relatively conserved. Approximately $40 \%$ of patients in both groups had BCLC-B (intermediate stage) $\mathrm{HCC}$, although the percentage of patients with BCLC-B2 stage HCC was higher in the combination group. Most patients had prior treatments, including TACE. The characteristics of patients in the monotherapy and combination therapy groups did not differ significantly (Table 1). In all, 14 patients with MVI, whose disease was deemed uncontrollable by sorafenib monotherapy, were administered combination of sorafenib and HAIC, whereas 13 patients showing progression of intrahepatic lesions were administered combination of sorafenib and TACE (Figure 1). Of the patients who received TACE plus sorafenib, five, who did not achieve stable disease, were switched to HAIC plus sorafenib.

\section{Sorafenib treatment}

The details of sorafenib treatment are given in Table 2. The proportion of patients requiring sorafenib dose reductions was similar in the two groups. However, the duration of sorafenib treatment was significantly shorter in the monotherapy group. Evaluation of combination therapy showed that 13 (59.1\%) patients received TACE and 14 (68.4\%) received HAIC. The median number of TACE sessions per patient was 4 (range 1-8), and the median number of HAIC sessions per patient was 6 (range $2-8$ ). Five patients who were refractory to sorafenib plus TACE (median 2 sessions, range 1-2) were subsequently treated with sorafenib plus HAIC (median 6 sessions, range 4-8). Sorafenib was discontinued due to disease progression in about half of the patients. There were no significant between-group differences in adverse events causing sorafenib discontinuation and no significant betweengroup differences in antitumor effects. However, the objective response rates (complete response+partial response) in 
Table I Characteristics of patients

\begin{tabular}{|c|c|c|c|}
\hline Factors & Sorafenib & Combination Tx & $p$-value \\
\hline Gender (male/female) & $50 / 8$ & $19 / 3$ & 0.985 \\
\hline Age (years) & $68(37-83)$ & $67.5(53-82)$ & 0.564 \\
\hline Etiology (HCV/HBV/Others) & $20 / 15 / 23$ & $6 / 7 / 9$ & 0.767 \\
\hline ECOG PS $(0,1 / 2)$ & $54 / 4$ & $21 / 1$ & 0.579 \\
\hline Child (A/B/C) & $43 / 15 / 0$ & $20 / 2 / 0$ & 0.102 \\
\hline Serum Alb (g/dL) & $3.6(2.2-4.5)$ & $3.9(2.2-4.3)$ & 0.202 \\
\hline Serum T-Bil (mg/dL) & $0.75(0.3-2.8)$ & $0.65(0.3-1.9)$ & 0.139 \\
\hline ALBI grade $(1 / 2 / 3)$ & $49 / 9 / 0$ & $20 / 2 / 0$ & 0.366 \\
\hline $\mathrm{Plt}\left(\times 10^{4} / \mathrm{mL}\right)$ & $12.7(6.9-58.5)$ & I $4.6(7.0-24.1)$ & 0.880 \\
\hline $\mathrm{BCLC}_{\text {stage }}$ (BI/B2/B3/C) & $15 / 4 / 0 / 39$ & $0 / 8 / 0 / 14$ & $0.526^{\mathrm{b}}$ \\
\hline Max tumor size $(\mathrm{cm})$ & $5.0(2.0-15.0)$ & $6.0(3.0-20.0)$ & 0.595 \\
\hline Tumor number & $4(1-20)$ & $6(1-20)$ & 0.490 \\
\hline EHM (yes/no) & $18 / 40$ & $5 / 17$ & 0.464 \\
\hline MVI (yes/no) & $30 / 28$ & $14 / 8$ & 0.241 \\
\hline Serum AFP $(\mathrm{ng} / \mathrm{mL})$ & $43.2(1.3-1,200,000)$ & $39.6(4.5-97,480)$ & 0.923 \\
\hline Serum DCP $(\mathrm{mAU} / \mathrm{mL})$ & $898(4.0-220,192)$ & $172(|9.0-7|, 386)$ & 0.165 \\
\hline Prior treatment (yes/no) & $49 / 9$ & $18 / 4$ & 0.509 \\
\hline Prior TACE number & $2(0-8)$ & $2(0-4)$ & 0.370 \\
\hline
\end{tabular}

Notes: Results are reported as number or as median (range). ${ }^{\mathrm{B} C L C}$-B subclassification according to Kinki criteria. ${ }^{\mathrm{b}} \mathrm{Comparison}$ between $\mathrm{BCLC}-\mathrm{B}$ and $\mathrm{BCLC}-\mathrm{C}$.

Abbreviations: Tx, therapy; HCV, hepatitis C virus; HBV, hepatitis B virus; ECOG, Eastern Cooperative Oncology Group; PS, performance status; Alb, albumin; T-Bil, total bilirubin; ALBI, albumin-bilirubin; Plt, platelet; BCLC, Barcelona clinical liver cancer; EHM, extrahepatic metastasis; MVI, major vascular invasion; AFP, alpha-fetoprotein; DCP, des-gamma-carboxy prothrombin; TACE, transarterial chemoembolization.

the monotherapy and combination therapy groups were $9.3 \%$ $(5 / 54)$ and $13.6 \%(3 / 22)$, respectively, whereas the disease control rate was significantly higher in the combination therapy group than in the monotherapy group $(86.3 \%$ vs $51.7 \%, p=0.01)$. Although the proportion of patients who were treated after sorafenib discontinuation did not differ significantly in the two groups, the percentage of patients who received only palliative care was frequently higher in the combination group. Combination therapy was also associated with higher rates of hepatotoxic and hematologic adverse

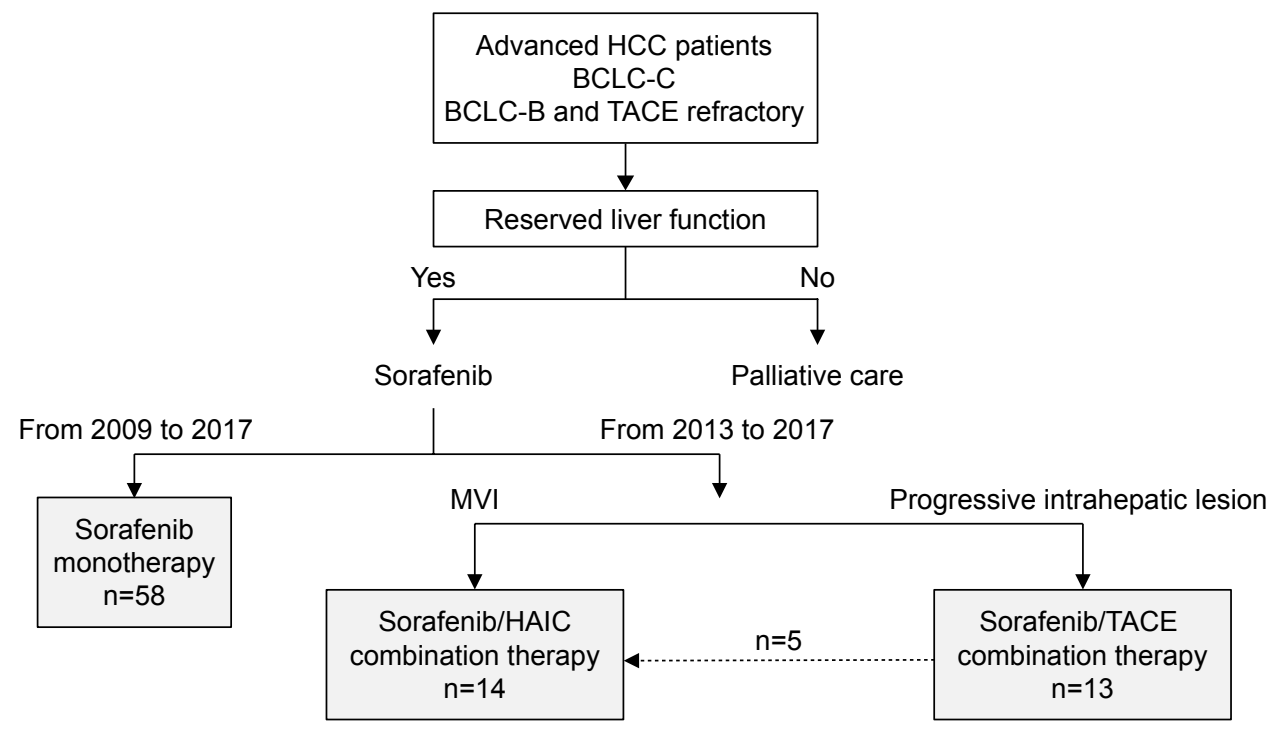

Figure I Flow chart of the present study.

Notes: Advanced HCC patients with BCLC-C or TACE-refractory BCLC-B and with reserved liver function were enrolled. Patients with intrahepatic lesions regarded as prognostic were included in the combination therapy group.

Abbreviations: HCC, hepatocellular carcinoma; BCLC, Barcelona clinical liver cancer; TACE, transarterial chemoembolization; MVI, major vascular invasion; HAIC, hepatic arterial infusion chemotherapy. 
Table 2 Sorafenib treatment parameters

\begin{tabular}{|l|l|l|l|}
\hline & Sorafenib $(\mathbf{n}=\mathbf{5 8})$ & Combination Tx $(\mathbf{n}=\mathbf{2 2})$ & $\mathbf{p}$-value \\
\hline Starting dose $800 \mathrm{mg} /$ day & $52(89.6)$ & $21(95.4)$ & 0.37 \\
\hline Duration of sorafenib (days) & 66 & 367 & $<0.00 \mathrm{I}$ \\
\hline Average dose (mg/day) & 495 & 488 & 0.86 \\
\hline Combination with TACE & 0 & $13(59.1)$ & $\mathrm{NE}$ \\
\hline Combination with HAIC & 0 & $14(63.6)$ & $\mathrm{NE}$ \\
\hline Cessation of sorafenib & $52(89.6)$ & $19(86.4)$ & 0.47 \\
\hline Disease progression & $27(51.9)$ & $13(68.4)$ & 0.16 \\
\hline Adverse events & $25(48.1)$ & $6(3 \mathrm{I} .6)$ & 0.17 \\
\hline Tumor response: CR/PR/SD/PD/NE & $\mathrm{I} / 4 / 25 / 24 / 4$ & $0 / 3 / 16 / 3 / 0$ & $\mathrm{NE}$ \\
\hline Objective response rate & $5(9.3)$ & $3(\mathrm{I3.6})$ & 0.42 \\
\hline DCR & $30(5 \mathrm{I} .7)$ & $19(86.3)$ & $0.0 \mathrm{I}$ \\
\hline Posttreatment (present) & $32(55.1)$ & $8(36.3)$ & $0.1 \mathrm{I}$ \\
\hline
\end{tabular}

Note: Results reported as number or number (\%).

Abbreviations: Tx, therapy; TACE, transarterial chemoembolization; HAIC, hepatic arterial infusion chemotherapy; CR, complete response; PR, partial response; SD, stable disease; PD, progressive disease; NE, not examined; DCR, disease control rate.

events (Table 3). However, the percentage of patients who discontinued sorafenib due to adverse effects was similar in the two groups (Table 2). These results showed that the combination therapy was tolerable and could prolong the duration of nonprogression of HCC.

\section{PFS and OS}

Evaluation of survival outcomes after sorafenib administration in all patients showed that median survival time (MST) was 94 days (95\% CI: 73.8-114.2 days) for PFS and 550 days (95\% CI: 352.6-747.4 days) for OS. OS in patients with BCLC-B tended to be longer than in those with BCLC-C (MST: 869 vs 477 days, respectively). OS in our BCLC-C cohort was longer than that in a previous study (11 months [330 days], 95\% CI 6-14 months). ${ }^{6}$ PFS was significantly longer in the combination therapy group than in the sorafenib monotherapy group ( 180 vs 85 days, $p=0.045$; Figure $2 \mathrm{~A}$ ) but did not differ significantly in patients with BCLC-B ( 120 vs 180 days, $p=0.896)$ and BCLC-C ( 180 vs 270 days, $p=0.149$ ) treated with TACE plus sorafenib and HAIC plus sorafenib. Median OS was significantly longer in the combination therapy group than in the sorafenib monotherapy group ( 983 vs 452 days, $p=0.004$; Figure 2B) but did not differ significantly in patients with BCLC-B

Table 3 Adverse events of sorafenib treatment

\begin{tabular}{|c|c|c|c|c|c|}
\hline & \multicolumn{2}{|c|}{ Sorafenib $(n=58)$} & \multicolumn{2}{|c|}{ Combination Tx $(n=22)$} & \multirow[t]{2}{*}{$p$-value } \\
\hline & All grade & Grade 3/4 & All grade & Grade 3/4 & \\
\hline Diarrhea & $9(15.5)$ & 0 & $4(18.2)$ & 0 & 0.51 \\
\hline HFSR & $39(67.2)$ & $7(12.1)$ & $15(68.2)$ & $3(13.6)$ & 0.58 \\
\hline Fatigue & $7(12.1)$ & 0 & $3(13.6)$ & 0 & 0.56 \\
\hline Nausea/vomiting & $3(5.2)$ & 0 & $2(9.1)$ & 0 & 0.42 \\
\hline Anorexia & $8(13.8)$ & 0 & $3(13.6)$ & 0 & 0.63 \\
\hline Hypertension & $12(20.6)$ & $\mathrm{I}(\mathrm{I} .7)$ & $3(13.6)$ & 0 & 0.35 \\
\hline Alopecia & $9(15.5)$ & 0 & $4(18.2)$ & 0 & 0.51 \\
\hline Elevated ALT & $7(12.1)$ & I (I.7) & $9(40.9)$ & $2(9.1)$ & 0.01 \\
\hline Elevated Amy & I5 (25.9) & $2(3.4)$ & $4(18.2)$ & $\mathrm{I}(4.5)$ & 0.34 \\
\hline Hyperbilirubinemia & $3(5.2)$ & 0 & $2(9.1)$ & 0 & 0.42 \\
\hline Rash & $5(8.6)$ & $5(8.6)$ & $2(9.1)$ & $2(9.1)$ & 0.63 \\
\hline Leukocytopenia & $4(6.9)$ & $\mathrm{I}(\mathrm{I} .7)$ & $4(18.2)$ & I (4.5) & 0.14 \\
\hline Thrombocytopenia & $10(17.2)$ & $2(3.4)$ & $7(3 \mid .8)$ & $\mathrm{I}(4.5)$ & 0.13 \\
\hline
\end{tabular}

Note: Results reported as number (\%).

Abbreviations: Tx, therapy; HFSR, hand-foot skin reaction; ALT, alanine aminotransferase; Amy, amylase. 


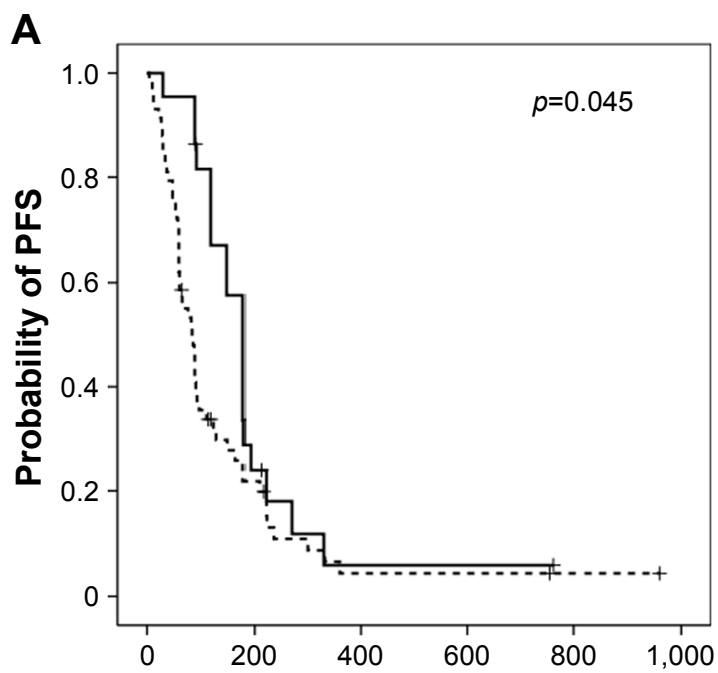

Days after sorafenib administration

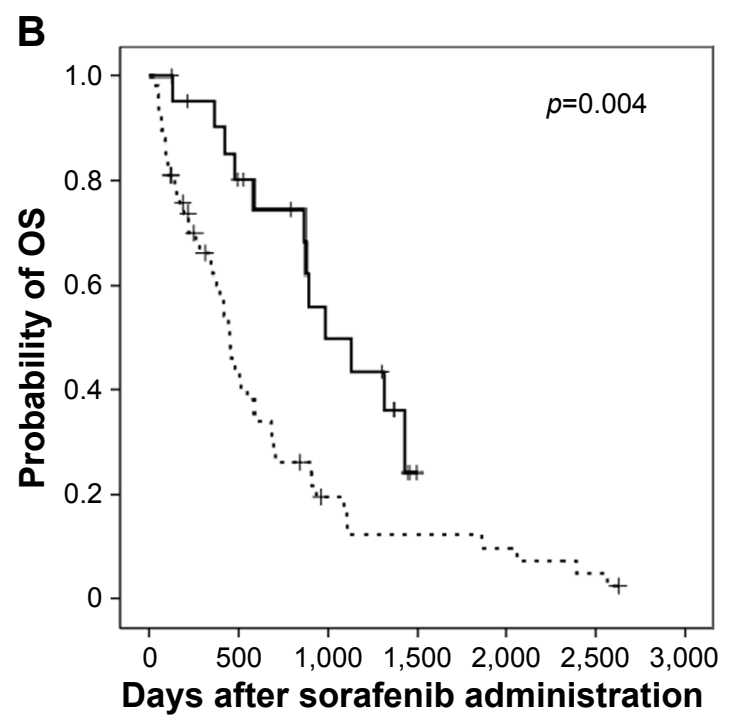

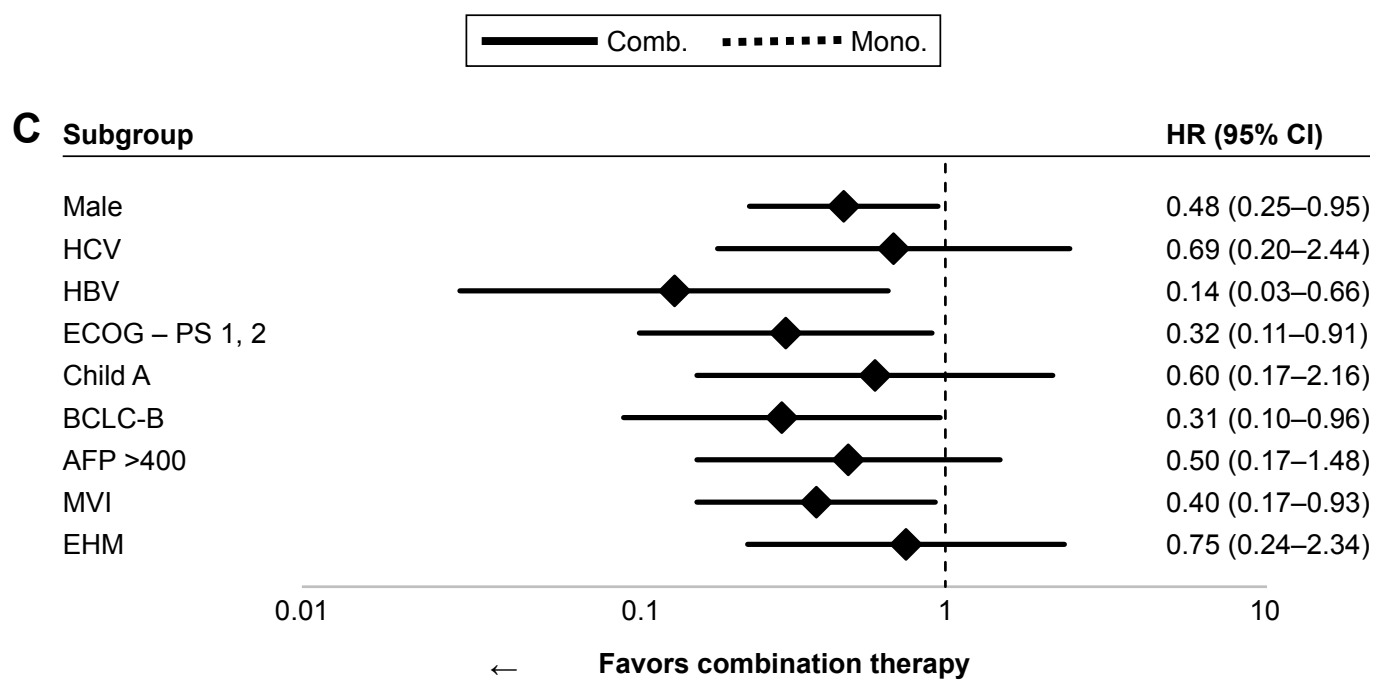

Figure 2 Kaplan-Meier analyses of (A) PFS and (B) OS in groups of patients administered combination therapy (Comb.; $\mathrm{n}=22)$ and sorafenib monotherapy (Mono.; $\mathrm{n}=58$ ). Between-group differences were analyzed by log-rank tests. (C) Forest-plot analysis.

Abbreviations: PFS, progression-free survival; OS, overall survival; HCV, hepatitis C virus; HBV, hepatitis B virus; ECOG, Eastern Cooperative Oncology Group; PS, performance status; BCLC, Barcelona clinical liver cancer; AFP, alpha-fetoprotein; MVI, major vascular invasion; EHM, extrahepatic metastasis.

(not reached vs 850 days, $p=0.135)$ and BCLC-C (1,180 vs 580 days, $p=0.245)$ treated with TACE plus sorafenib and HAIC plus sorafenib. The duration of sorafenib treatment was significantly longer in the combination therapy than in the monotherapy (Table 2). These results suggested that combination therapy could provide better disease control than monotherapy, prolonging sorafenib administration and OS.

\section{Survival benefits of combination therapy}

Univariate analysis revealed that $\mathrm{HCV}$ infection, BCLC-B, disease control, and combination therapy were associated with prolonged survival, whereas HBV infection, decreased PS, increased alpha-fetoprotein (AFP) (>400 ng/mL), and the presence of MVI were associated with shorter survival (Table 4). Multivariate analysis showed that HCV infection, increased AFP (>400 ng/mL), disease control, and combination therapy were independently prognostic of survival (Table 4). Cox regression analysis to determine patients who could benefit from combination therapy showed that HBV infection (HR 0.14; 95\% CI: 0.03-0.66), the presence of symptoms (HR 0.32, 95\% CI: 0.11-0.91), BCLC-B (HR 0.31, 95\% CI: 0.10-0.96), and MVI (HR 0.40, 95\% CI: 0.17-0.93) were associated with better prognosis with combination therapy (Figure 2C). These results indicate that combination therapy provides survival benefits to patients whose disease is difficult to control with sorafenib monotherapy. 
Table 4 Univariate and multivariate analyses of factors associated with OS

\begin{tabular}{|c|c|c|c|c|c|c|}
\hline \multirow[t]{2}{*}{ Variables } & \multicolumn{3}{|c|}{ Univariate Cox analysis } & \multicolumn{3}{|c|}{ Multivariate Cox analysis } \\
\hline & HR & $95 \% \mathrm{Cl}$ & p-value & HR & $95 \% \mathrm{Cl}$ & $p$-value \\
\hline Male & 1.36 & $0.64-2.88$ & 0.42 & & & \\
\hline HCV infection & 0.47 & $0.26-0.86$ & 0.01 & 0.39 & $0.18-0.82$ & 0.01 \\
\hline HBV infection & 1.86 & $1.05-3.29$ & 0.03 & 1.42 & $0.72-2.80$ & 0.31 \\
\hline ECOG PS & 1.87 & I. $17-2.97$ & $<0.01$ & 1.65 & $0.96-2.87$ & 0.07 \\
\hline Child A & 0.62 & $0.32-|.2|$ & 0.16 & & & \\
\hline ALBI grade 2 & 1.47 & $0.72-3.00$ & 0.29 & & & \\
\hline BCLC-B & 0.60 & $0.35-1.05$ & 0.07 & 1.42 & $0.59-3.40$ & 0.43 \\
\hline AFP $>400 \mathrm{ng} / \mathrm{mL}$ & 2.26 & $|.34-3.8|$ & $<0.01$ & 2.15 & $1.23-3.74$ & $<0.01$ \\
\hline $\mathrm{MVI+}$ & 1.89 & $1.13-3.16$ & 0.02 & 1.68 & $0.76-3.71$ & 0.20 \\
\hline EHM+ & 0.99 & $0.58-1.72$ & 0.99 & & & \\
\hline DCR+ & 0.49 & $0.29-0.82$ & $<0.01$ & 0.51 & $0.29-0.92$ & 0.03 \\
\hline Combination Tx & 0.40 & $0.21-0.77$ & $<0.01$ & 0.34 & $0.16-0.71$ & $<0.01$ \\
\hline
\end{tabular}

Abbreviations: OS, overall survival; HCV, hepatitis C virus; HBV, hepatitis B virus; ECOG, Eastern Cooperative Oncology Group; PS, performance status; ALBI, albuminbilirubin; BCLC, Barcelona clinic liver cancer; AFP, alpha-fetoprotein; MVI, major vascular invasion; EHM, extrahepatic metastasis; DCR, disease control rate; Tx, therapy.

\section{Discussion}

The present study showed that combination therapy with sorafenib plus hepatic arterial treatment, TACE or HAIC, was effective and tolerable in patients with advanced HCC. Sorafenib has been reported to be insufficient to treat HCC patients with severe portal vein tumor thrombi ${ }^{29}$ and less effective for patients infected with HBV. ${ }^{30}$ Combination therapy may be effective in patients who are likely to be refractory to sorafenib, as it may counter these deficiencies of sorafenib. In a large-scale global postmarketing survey (global investigation of therapeutic decisions in HCC and of its treatment with sorafenib, GIDEON), 325 patients, or $10.1 \%$ of all patients, administered TACE and sorafenib concomitantly showed longer OS than patients administered non-concomitant TACE (21.6 vs 9.7 months). ${ }^{31}$ Moreover, the median duration of sorafenib treatment was longer in patients with concomitant TACE (36.4 vs 13.1 weeks), ${ }^{31}$ similar to the findings of the present study. Sorafenib continued to be effective even after radiologic confirmation of progressive disease. ${ }^{32}$ Prolonged sorafenib treatment is considered useful in patients with advanced HCC. For example, a comparative study that included propensity score analysis found that TTP was longer in patients treated with TACE plus sorafenib than with sorafenib alone. ${ }^{33}$ Although their comparative effects on OS were unclear, combination therapy was found to prolong OS in a subgroup of patients with vascular invasion, ${ }^{33}$ similar to the results of the present study. Thus, sorafenib combined with TACE may be useful in patients with MVI or in those who may be refractory to sorafenib monotherapy, by improving disease control and prolonging the duration of sorafenib administration.

The present study also found that combination therapy was favorable in patients with BCLC-B. Many patients with BCLC-B2 were included in the combination therapy group, as combination therapy was considered for patients showing rapid tumor progression. Shorter TTP after TACE was shown to be closely associated with poor prognosis..$^{34,35}$ Sorafenib-based combination therapy may therefore be a treatment option in patients with aggressive BCLC-B2 HCC.

TKIs and hepatic arterial therapy may show positive synergistic effects. TKIs can suppress the increased expression of VEGF induced after arterial embolization, ${ }^{13}$ and tumor vasculature can be normalized by anti-VEGF drugs. ${ }^{36,37}$ However, four randomized studies found no synergistic effects between TKIs and hepatic arterial therapy on survival outcomes, especially on OS, but did show an increase in adverse events. ${ }^{38-41}$ In these studies, TACE was administered every 4-6 weeks until complete tumor necrosis. ${ }^{38-40}$ This schedule of TACE may have resulted in a higher incidence of severe adverse events than on-demand TACE. ${ }^{42}$ Metaanalyses confirmed that, compared with TACE monotherapy, combination therapy with sorafenib plus TACE improved TTP, ${ }^{14-17}$ but the effects on OS are unclear. ${ }^{18,19}$ A recent review suggested that the timing of sorafenib administration, the low dose and short duration of sorafenib treatment, the adverse effects of scheduled TACE, and the definition of progression based on RESIST criteria are not adequate in trials of TACE combination. ${ }^{43}$ A randomized Phase II trial 
assessing the effectiveness of the combination of sorafenib and TACE, administered on demand not on a fixed schedule (TACTICS trial), clearly showed that this combination had positive effects on survival outcomes. ${ }^{44}$ These findings suggest that the effectiveness of combination therapy may have been masked by adverse events.

The potential benefits of TACE in patients with advanced HCC have been described. A large case-control study showed that TACE was safe and effective in patients with MVI. ${ }^{45}$ Moreover, intrahepatic control by TACE was shown to benefit patients with EHM. ${ }^{46}$ Potential adverse effects should be carefully considered in individual patients prior to initiating combination therapy.

HAIC is a treatment option for advanced HCC, especially in Japan, inducing tumor shrinkage or regression of vascular invasion. Its concept is based on increased local concentration of anticancer drugs in the tumor and reduced systemic distribution of these drugs. ${ }^{47}$ Comparative studies showed that HAIC had more favorable treatment outcomes than sorafenib. ${ }^{48,49}$ In a recent Phase III trial (SILIUS trial), the combination effect of sorafenib and HAIC was shown to have positive effects in patients with MVI. ${ }^{22} \mathrm{HAIC}$ is not a standard treatment modality for advanced HCC except in Japan. However, there is no established therapeutic modality for advanced HCC with severe MVI. On-demand HAIC in combination with sorafenib may be a therapeutic option for these patients. Combinations of sorafenib and these hepatic arterial therapies shown superior for tumor control may be beneficial if adverse events are better tolerated.

The present study had several limitations. First, this was a retrospective study, and the number of patients was relatively small. Biases due to the nonmatched and unbalanced number of patients in the two groups, as well as treatment selection and confounding variables, cannot be ruled out. However, the major demographic and clinical characteristics of the two groups were almost identical, and our results were consistent with those of previous studies. ${ }^{20-22,44}$ Further studies with larger cohorts are desired. Second, this study evaluated two combinations, sorafenib plus TACE and sorafenib plus HAIC. These two modalities have different characteristics, and HAIC is not a recommended standard therapy except in Japan. Combining these groups may have affected the interpretation of the results. However, the antitumor effects of these combinations and the survival times of the two subgroups did not differ significantly. Furthermore, the combination therapies were administered to patients with extrahepatic metastases if they were likely to enhance control of intrahepatic lesions, as the present study addressed the use of combination therapies to treat intrahepatic lesions. Further investigations of each modality independently and clarifications of their indications are required.

In addition, new treatment options may be available for patients with advanced HCC in the near future. Regorafenib was shown to provide survival benefit in patients with advanced HCC who were refractory to sorafenib (RESORCE trial). ${ }^{11}$ However, the duration of sorafenib treatment was longer than that in a second clinical trial (GIDEON trial; 7.8 vs 3.8 months). ${ }^{11,30}$ That is, some ingenuity might be required to prolong the duration of sorafenib treatment in clinical settings. The present study also found that hepatotoxicity was frequent in the combination therapy group. Furthermore, repeated administration of TACE was found to be associated with a decline in hepatic function. ${ }^{50}$ Although this decline was not considered problematic in the present study of combination therapies, hepatic function should be maintained in patients with advanced HCC, allowing secondline treatments or alternative therapies to be considered. Recently, nivolumab, a programmed cell death protein-1 immune checkpoint inhibitor, was reported to show higher tumor response and lower adverse event rates. ${ }^{51}$ Furthermore, combination therapies with TKIs and such immune checkpoint inhibitors may extend survival in patients with advanced HCC. ${ }^{52}$ Studies are needed to optimize use of these new drugs and combination therapies including new agents.

\section{Conclusion}

This study showed that the combination of sorafenib and hepatic arterial treatment was effective and tolerable in patients with advanced HCC, even in those with MVI or HBV infection who might be refractory to sorafenib monotherapy. Combining treatment modalities that are superior in controlling tumors might lead to better disease control, allowing prolonged administration of sorafenib. Prolonged treatment duration may further improve disease control. Further investigations, including the control of adverse events and optimal agents combined with TKIs, are required.

\section{Acknowledgment}

This work was partly supported by JSPS KAKENHI Grant Number JP17K09410.

\section{Disclosure}

The authors report no conflicts of interest in this work.

\section{References}

1. Llovet JM, Burroughs A, Bruix J. Hepatocellular carcinoma. Lancet. 2003;362:1907-1917. doi:10.1016/S0140-6736(03)14964-1 
2. Forner A, Llovet JM, Bruix J. Hepatocellular carcinoma. Lancet. 2012; 379:1245-1255. doi:10.1016/S0140-6736(11)61347-0

3. Kumar A, Srivastava DN, Chau TT, et al. Inoperable hepatocellular carcinoma: transarterial 188Re HDD-labeled iodized oil for treatmentprospective multicenter clinical trial. Radiology. 2007;243:509-519. doi:10.1148/radiol.2432051246

4. Lo CM, Ngan H, Tso WK, et al. Randomized controlled trial of transarterial lipiodol chemoembolization for unresectable hepatocellular carcinoma. Hepatology. 2002;35:1164-1171. doi:10.1053/jhep.2002.33156

5. Llovet JM, Real MI, Montana X, et al. Arterial embolisation or chemoembolisation versus symptomatic treatment in patients with unresectable hepatocellular carcinoma: a randomised controlled trial Lancet. 2002;359:1734-1739. doi:10.1016/S0140-6736(02)08649-X

6. Bruix J, Sherman M; American Association for the Study of Liver Disease. Management of hepatocellular carcinoma: an update. Hepatology. 2011;53:1020-1022. doi:10.1002/hep.24199

7. Arizumi T, Ueshima K, Chishina H, et al. Validation of the criteria of transcatheter arterial chemoembolization failure or refractoriness in patients with advanced hepatocellular carcinoma proposed by the LCSGJ. Oncology. 2014;87(Suppl 1):32-36. doi:10.1159/000368143

8. Ogasawara S, Chiba T, Ooka Y, et al. Efficacy of sorafenib in intermediate-stage hepatocellular carcinoma patients refractory to transarterial chemoembolization. Oncology. 2014;87:330-341. doi:10.1159/000365993

9. Llovet JM, Ricci S, Mazzaferro V, et al. Sorafenib in advanced hepatocellular carcinoma. $N$ Engl J Med. 2008;359:378-390. doi:10.1056 NEJMoa0708857

10. Cheng AL, Kang YK, Chen Z, et al. Efficacy and safety of sorafenib in patients in the Asia-Pacific region with advanced hepatocellular carcinoma: a phase III randomised, double-blind, placebo-controlled trial. Lancet Oncol. 2009;10:25-34. doi:10.1016/S1470-2045(08)70285-7

11. Bruix J, Qin S, Merle P, et al. Regorafenib for patients with hepatocellular carcinoma who progressed on sorafenib treatment (RESORCE) a randomised, double-blind, placebo-controlled, phase 3 trial. Lancet 2017;389:56-66. doi:10.1016/S0140-6736(16)32453-9

12. Kudo M, Finn RS, Qin S, et al. Lenvatinib versus sorafenib in first-line treatment of patients with unresectable hepatocellular carcinoma: a randomised phase 3 non-inferiority trial. Lancet. 2018;391:1163-1173. doi:10.1016/S0140-6736(18)30207-1

13. Weintraub JL, Salem R. Treatment of hepatocellular carcinoma combining sorafenib and transarterial locoregional therapy: state of the science. J Vasc Interv Radiol. 2013;24:1123-1134. doi:10.1016/j. jvir.2013.01.494

14. Fu QH, Zhang Q, Bai XL, et al. Sorafenib enhances effects of transarterial chemoembolization for hepatocellular carcinoma: a systematic review and meta-analysis. J Cancer Res Clin Oncol. 2014;140: 1429-1440. doi:10.1007/s00432-014-1684-5

15. Zhang L, Hu P, Chen X, Bie P. Transarterial chemoembolization (TACE) plus sorafenib versus TACE for intermediate or advanced stage hepatocellular carcinoma: a meta-analysis. PLoS One. 2014;9:e100305. doi:10.1371/journal.pone.0100305

16. Zhang X, Wang K, Wang M, et al. Transarterial chemoembolization (TACE) combined with sorafenib versus TACE for hepatocellular carcinoma with portal vein tumor thrombus: a systematic review and metaanalysis. Oncotarget. 2017;8:29416-29427. doi:10.18632/oncotarget.15075

17. Cai R, Song R, Pang P, et al. Transcatheter arterial chemoembolization plus sorafenib versus transcatheter arterial chemoembolization alone to treat advanced hepatocellular carcinoma: a meta-analysis. BMC Cancer. 2017;17:714. doi:10.1186/s12885-017-3707-5

18. Liu L, Chen H, Wang M, et al. Combination therapy of sorafenib and TACE for unresectable HCC: a systematic review and meta-analysis. PLoS One. 2014;9:e91124. doi:10.1371/journal.pone.0091124

19. Zeng J, Lv L, Mei ZC. Efficacy and safety of transarterial chemoembolization plus sorafenib for early or intermediate stage hepatocellular carcinoma: a systematic review and meta-analysis of randomized controlled trials. Clin Res Hepatol Gastroenterol. 2016;40:688-697. doi:10.1016/j.clinre.2016.04.006
20. Ueshima K, Kudo M, Tanaka M, et al. Phase I/II study of sorafenib in combination with hepatic arterial infusion chemotherapy using low-dose cisplatin and 5-fluorouracil. Liver Cancer. 2015;4:263-273. doi:10.1159/000367751

21. Ikeda M, Shimizu S, Sato T, et al. Sorafenib plus hepatic arterial infusion chemotherapy with cisplatin versus sorafenib for advanced hepatocellular carcinoma: randomized phase II trial. Ann Oncol. 2016; 27:2090-2096. doi:10.1093/annonc/mdw323

22. Kudo M, Ueshima K, Yokosuka O, et al. Sorafenib plus low-dose cisplatin and fluorouracil hepatic arterial infusion chemotherapy versus sorafenib alone in patients with advanced hepatocellular carcinoma (SILIUS): a randomised, open label, phase 3 trial. Lancet Gastroenterol Hepatol. 2018;3:424-432. doi:10.1016/S2468-1253(18)30078-5

23. Johnson PJ, Berhane S, Kagebayashi C, et al. Assessment of liver function in patients with hepatocellular carcinoma: a new evidencebased approach - the ALBI grade. J Clin Oncol. 2015;33:550-558. doi:10.1200/JCO.2014.57.9151

24. Hiraoka A, Kumada T, Kudo M, et al. Albumin-bilirubin (ALBI) grade as part of the evidence-based clinical practice guideline for HCC of the Japan Society of Hepatology: a comparison with the liver damage and Child-Pugh classifications. Liver Cancer. 2017;6:204-215. doi: $10.1159 / 000452846$

25. Kudo M, Arizumi T, Ueshima K, et al. Subclassification of BCLC B stage hepatocellular carcinoma and treatment strategies: proposal of modified Bolondi's subclassification (Kinki criteria). Dig Dis. 2015;33:751-758. doi: $10.1159 / 000439290$

26. Kudo M, Matsui O, Izumi N, et al. JSH consensus-based clinical practice guidelines for the management of hepatocellular carcinoma: 2014 update by the Liver Cancer Study Group of Japan. Liver Cancer. 2014; 3:458-468. doi:10.1159/000343875

27. Tajiri K, Kawai K, Minemura M, et al. Neutrophil/lymphocyte ratio as a prognostic indicator of hepatic arterial infusion chemotherapy with arterial cisplatin plus continuous 5-fluorouracil. Hepatol Res. 2015;45: 755-763. doi:10.1111/hepr.12417

28. Lencioni R, Llovet JM. Modified RECIST (mRECIST) assessment for hepatocellular carcinoma. Semin Liver Dis. 2010;30:52-60. doi: $10.1055 / \mathrm{s}-0030-1247132$

29. Chan SL, Chong CC, Chan AW, et al. Management of hepatocellular carcinoma with portal vein tumor thrombosis: review and update at 2016 World J Gastroenterol.2016;22:7289-7300. doi:10.3748/wjg.v22.i32.7289

30. Jackson R, Psarelli EE, Berhane S, et al. Impact of viral status on survival in patients receiving sorafenib for advanced hepatocellular cancer: a meta-analysis of randomized phase III trials. J Clin Oncol. 2017;35:622-628. doi:10.1200/JCO.2016.69.5197

31. Geschwind JF, Kudo M, Marrero JA, et al. TACE treatment in patients with sorafenib-treated unresectable hepatocellular carcinoma in clinical practice: final analysis of GIDEON. Radiology. 2016;279:630-640. doi:10.1148/radiol.2015150667

32. Wada Y, Takami Y, Tateishi M, et al. The efficacy of continued sorafenib treatment after radiologic confirmation of progressive disease in patients with advanced hepatocellular carcinoma. PLoS One. 2016;11: e0146456. doi:10.1371/journal.pone. 0146456

33. Choi GH, Shim JH, Kim MJ, et al. Sorafenib alone versus sorafenib combined with transarterial chemoembolization for advanced-stage hepatocellular carcinoma: results of propensity score analyses. Radiology. 2013;269:603-611. doi:10.1148/radiol.13130150

34. Arizumi T, Minami T, Chishina H, et al. Time to transcatheter arterial chemoembolization refractoriness in patients with hepatocellular carcinoma in Kinki criteria stages B1 and B2. Dig Dis. 2017;35:589-597. doi: $10.1159 / 000480208$

35. Arizumi T, Ueshima K, Iwanishi M, et al. The overall survival of patients with hepatocellular carcinoma correlates with the newly defined time to progression after transarterial chemoembolization. Liver Cancer. 2017;6:227-235. doi:10.1159/000475777

36. Jain RK. Normalization of tumor vasculature: an emerging concept in antiangiogenic therapy. Science. 2005;307:58-62. doi:10.1126/ science. 1104819 
37. Kerbel RS. Antiangiogenic therapy: a universal chemosensitization strategy for cancer? Science. 2006;312:1171-1175. doi:10.1126/ science. 1125950

38. Sansonno D, Lauletta G, Russi S, Conteduca V, Sansonno L, Dammacco F. Transarterial chemoembolization plus sorafenib: a sequential therapeutic scheme for HCV-related intermediate-stage hepatocellular carcinoma: a randomized clinical trial. Oncologist. 2012;17:359-366. doi:10.1634/theoncologist.2011-0313

39. Lencioni R, Llovet JM, Han G, et al. Sorafenib or placebo plus TACE with doxorubicin-eluting beads for intermediate stage HCC: the SPACE trial. J Hepatol. 2016;64:1090-1098. doi:10.1016/j.jhep.2016.01.012

40. Hoffmann K, Ganten T, Gotthardtp D, et al. Impact of neo-adjuvant sorafenib treatment on liver transplantation in HCC patients - a prospective, randomized, double-blind, phase III trial. BMC Cancer. 2015;15: 392. doi:10.1186/s12885-015-1584-3

41. Kudo M, Imanaka K, Chida N, et al. Phase III study of sorafenib after transarterial chemoembolisation in Japanese and Korean patients with unresectable hepatocellular carcinoma. Eur J Cancer. 2011;47: 2117-2127. doi:10.1016/j.ejca.2011.05.007

42. Tavernier J, Fagnoni P, Chabrot P, et al. Comparison of two transarterial chemoembolization strategies for hepatocellular carcinoma. Anticancer Res. 2014;34:7247-7253.

43. Kudo M, Arizumi T. Transarterial chemoembolization in combination with a molecular targeted agent: lessons learned from negative trials (Post-TACE, BRISK-TA, SPACE, ORIENTAL, and TACE-2). Oncology. 2017;93(Suppl 1):127-134. doi:10.1159/000481243

44. Kudo M, Ueshima K, Ikeda M, et al. Randomized, open label, multicenter, phase II trial comparing transarterial chemoembolization (TACE) plus sorafenib with TACE alone in patients with hepatocellular carcinoma (HCC): TACTICS trial. J Clin Oncol. 2018;36(suppl4S; abstr 206).
45. Chung GE, Lee JH, Kim HY, et al. Transarterial chemoembolization can be safely performed in patients with hepatocellular carcinoma invading the main portal vein and may improve the overall survival. Radiology. 2011;258:627-634. doi:10.1148/radiol.10101058

46. Yoo DJ, Kim KM, Jin YJ, et al. Clinical outcome of 251 patients with extrahepatic metastasis at initial diagnosis of hepatocellular carcinoma: does transarterial chemoembolization improve survival in these patients? J Gastroenterol Hepatol. 2011;26:145-154. doi:10.1111/j.1440-1746.2010.06341.x

47. Kudo M. Treatment of advanced hepatocellular carcinoma with emphasis on hepatic arterial infusion chemotherapy and molecular targeted therapy. Liver Cancer. 2012;1:62-70. doi:10.1159/000342402

48. Hiramine Y, Uto H, Imamura Y, et al. Sorafenib and hepatic arterial infusion chemotherapy for unresectable advanced hepatocellular carcinoma: a comparative study. Exp Ther Med. 2011;2:433-441. doi:10.3892/etm.2011.237

49. Song DS, Song MJ, Bae SH, et al. A comparative study between sorafenib and hepatic arterial infusion chemotherapy for advanced hepatocellular carcinoma with portal vein tumor thrombosis. $J$ Gastroenterol. 2015;50:445-454. doi:10.1007/s00535-014-0978-3

50. Hiraoka A, Kumada T, Kudo M, et al. Hepatic function during repeated TACE procedures and prognosis after introducing sorafenib in patients with unresectable hepatocellular carcinoma: multicenter analysis. Dig Dis. 2017;35:602-610. doi:10.1159/000480256

51. El-Khoueiry AB, Sangro B, Yau T, et al. Nivolumab in patients with advanced hepatocellular carcinoma (CheckMate 040): an open-label, non-comparative, phase 1/2 dose escalation and expansion trial. Lancet. 2017;389:2492-2502. doi:10.1016/S0140-6736(17)31046-2

52. Kudo M. Systemic therapy for hepatocellular carcinoma: 2017 update. Oncology. 2017;93(Supp1 1):135-146. doi:10.1159/000481244
OncoTargets and Therapy

\section{Publish your work in this journal}

OncoTargets and Therapy is an international, peer-reviewed, open access journal focusing on the pathological basis of all cancers, potential targets for therapy and treatment protocols employed to improve the management of cancer patients. The journal also focuses on the impact of management programs and new therapeutic agents and protocols on

\section{Dovepress}

patient perspectives such as quality of life, adherence and satisfaction The manuscript management system is completely online and includes a very quick and fair peer-review system, which is all easy to use. Visit http://www.dovepress.com/testimonials.php to read real quotes from published authors. 